\title{
Quantum Information Transfer and Entanglement with SQUID Qubits in Cavity QED: A Dark-State Scheme with Tolerance for Nonuniform Device Parameter
}

\author{
Chui-Ping Yang, ${ }^{1,2}$ Shih-I Chu, ${ }^{2}$ and Siyuan Han $^{1}$ \\ ${ }^{1}$ Department of Physics and Astronomy, University of Kansas, Lawrence, Kansas 66045, USA \\ ${ }^{2}$ Department of Chemistry, University of Kansas, and Kansas Center for Advanced Scientific Computing, \\ Lawrence, Kansas 66045, USA
}

(Received 25 December 2002; published 18 March 2004)

\begin{abstract}
We investigate the experimental feasibility of realizing quantum information transfer (QIT) and entanglement with SQUID qubits in a microwave cavity via dark states. Realistic system parameters are presented. Our results show that QIT and entanglement with two-SQUID qubits can be achieved with a high fidelity. The present scheme is tolerant to device parameter nonuniformity. We also show that the strong coupling limit can be achieved with SQUID qubits in a microwave cavity. Thus, cavitySQUID systems provide a new way for production of nonclassical microwave source and quantum communication.
\end{abstract}

Superconducting devices including single Cooper pair boxes, Josephson junctions, and superconducting quantum interference devices (SQUIDs) [1-10] have appeared to be among the most promising candidates for quantum information processing. Superconducting qubits are relatively easy to scale up and have been demonstrated to have a long decoherence time [10-12]. In past years, many methods for demonstrating macroscopic coherence in SQUIDs [5] or performing a single-"SQUID qubit" operation [6-10] have been presented. Recently, spectroscopy evidence of entanglement in two charge qubits or Josephson junctions has also been reported $[13,14]$. However, how to obtain a two-SQUID qubit operation, which is the key ingredient for any quantum computing algorithms, has not been thoroughly investigated.

In this Letter, we discuss how quantum information transfer (QIT) and entanglement can be achieved with two-SQUID qubits in cavity QED via dark states, and then we give a detailed analysis on the experimental feasibility. This proposal has advantages: (i) Because the population in the level $|a\rangle$ (defined below) is minimized, spontaneous emission from this level is greatly suppressed and thus QIT and entanglement can be realized with a high fidelity. (ii) No tunneling between the SQUID qubit levels $|0\rangle$ and $|1\rangle$ is required; therefore decay from the level $|1\rangle$ can be made negligibly small during the operation, via adjusting the potential barrier. (iii) Since the cavity mode acts as a "bus" and can mediate long range, fast interaction between distant SQUID qubits, the cavity-based scheme is simpler than the noncavity schemes where significant resources may be needed to couple distant SQUID qubits. (iv) SQUIDs are sensitive to environment. By placing SQUIDs into a superconducting cavity, decoherence caused by external environment is greatly suppressed because the cavity can be doubled as a magnetic shield for SQUIDs. (v) Level structure of each individual SQUID qubit can be adjusted by either design variations and/or changing local bias field. Hence, coupling between microwave pulse and any particular SQUID qubit can be obtained selectively via frequency matching. (vi) The position of SQUID qubits in a cavity is fixed while for cavity-atom systems it remains a significant technical challenge to control the center of mass motion of a neutral atom.

The SQUIDs considered in this Letter are rf SQUIDs each consisting of a Josephson tunnel junction in a superconducting loop (typical size is $\sim 10-100 \mu \mathrm{m}$ ). The Hamiltonian of an rf SQUID (with junction capacitance $C$ and loop inductance $L$ ) can be written as [7]

$$
H_{s}=\frac{Q^{2}}{2 C}+\frac{\left(\Phi-\Phi_{x}\right)^{2}}{2 L}-E_{J} \cos \left(2 \pi \frac{\Phi}{\Phi_{0}}\right)
$$

where $\Phi$ is the magnetic flux threading the ring, $Q$ is the total charge on the capacitor, $\Phi_{x}$ is the external flux applied to the ring, and $E_{J} \equiv I_{c} \Phi_{0} / 2 \pi$ is the maximum Josephson coupling energy $\left(I_{c}\right.$ is the critical current of the junction and $\Phi_{0}=h / 2 e$ is the flux quantum).

Let us consider two SQUIDs I and II coupled to a single-mode microwave cavity field. Each SQUID qubit has a $\Lambda$-type configuration formed by the two lowest levels and an excited level, denoted by $|0\rangle,|1\rangle$, and $|a\rangle$ with energy eigenvalues $E_{0}, E_{1}$, and $E_{a}$, respectively [Figs. 1(a) and 1(b)]. The magnetic component of the microwave pulse applied to the SQUID $l$ is given by $\mathbf{B}_{\mu w l}(\mathbf{r}, t)=\tilde{\mathbf{B}}_{\mu w l}(\mathbf{r}, t) \cos 2 \pi \nu_{\mu w l} t(l=\mathrm{I}, \mathrm{II})$. Under the condition $D \gg d$ (where $D$ is the distance between the two SQUIDs and $d$ is the linear dimension of each SQUID), direct coupling between the two SQUIDs is negligible. This scheme does not require identical qubits because the level spacings $\Delta E_{a 0}=E_{a}-E_{0}$ of the two qubits can always be set to equal by adjusting flux bias while the difference in the level spacings $\Delta E_{a 1}=E_{a}-$ $E_{1}$ makes it straightforward to address each qubit using 


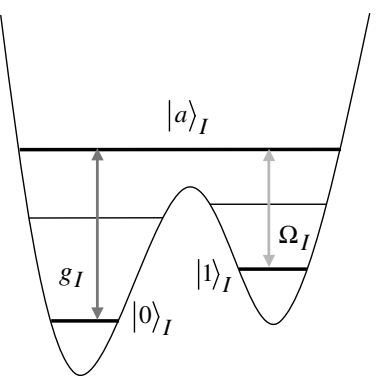

(a)

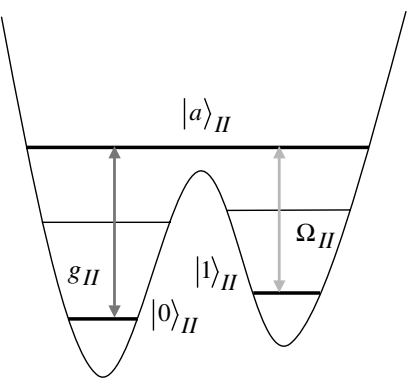

(b) (c)

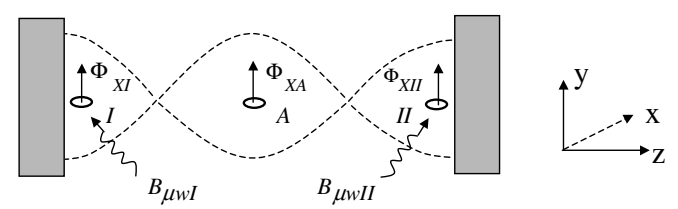

FIG. 1. (a) and (b) represent level diagrams of two nonidentical SQUIDs (I, II), respectively. The difference between $g_{\text {I }}$ and $g_{\text {II }}$ is due to device parameter nonuniformity or not exact placement of each SQUID qubit in a cavity. (c) Schematic illustration for two SQUIDs (I, II) and an auxiliary SQUID (A) in a standing-wave cavity. $\mathbf{B}_{c}, \mathbf{B}_{\mu w \mathrm{I}}$, and $\mathbf{B}_{\mu w \mathrm{II}}$ are in the $Y$ direction. SQUIDs are placed in the $X-Z$ plane. The auxiliary SQUID is used as a photon detector only in entanglement preparation.

different microwave frequencies. We can show that when the cavity field is resonant with the $|0\rangle \leftrightarrow|a\rangle$ transition of each SQUID and when the two pulses are tuned to the $|1\rangle \leftrightarrow|a\rangle$ transition of their respective SQUIDs, the interaction Hamiltonian of the system in the interaction picture, after the rotating-wave approximation, can be written as

$$
H=\hbar \sum_{l=\mathrm{I}, \mathrm{II}}\left[g_{l} c|a\rangle_{l}\left\langle 0\left|+\Omega_{l}(t)\right| a\right\rangle_{l}\langle 1|\right]+\text { H.c., }
$$

where $c^{+}$and $c$ are the photon creation and annihilation operators of the cavity mode with frequency $\nu_{c}=$ $\omega_{c} /(2 \pi), g_{l}$ is the coupling constant between the cavity mode and the $|0\rangle \leftrightarrow|a\rangle$ transition of the SQUID $l$, and $\Omega_{l}(t)$ is the Rabi frequency for the $|1\rangle \leftrightarrow|a\rangle$ transition of the SQUID $l$. The expressions of $g_{l}$ and $\Omega_{l}$ are given, respectively, by [15]

$$
\begin{aligned}
g_{l} & =\frac{1}{L_{l}} \sqrt{\frac{\omega_{c}}{2 \mu_{0} \hbar}}\langle 0|\Phi| a\rangle_{l} \int_{S_{l}} \mathbf{B}_{c}^{l}(\mathbf{r}) \cdot d \mathbf{S}, \\
\Omega_{l}(t) & =\frac{1}{2 L_{l} \hbar}\langle 1|\Phi| a\rangle_{l} \int_{S_{l}} \tilde{\mathbf{B}}_{\mu w l}(\mathbf{r}, \mathbf{t}) \cdot d \mathbf{S},
\end{aligned}
$$

where $S_{l}$ is any surface bounded by the ring of the SQUID $l$ and $\mathbf{B}_{c}^{l}(\mathbf{r})$ is the magnetic component of the normal mode of the cavity in the superconducting loop of the SQUID $l$. For a standing-wave cavity, one has $\mathbf{B}_{c}(z)=$ $\mu_{0} \sqrt{2 / V} \operatorname{cosk} z$ ( $\mathrm{k}$ is the wave number, $V$ and $z$ are the cavity volume and the cavity axis, respectively).
It is easy to verify that the following two states

$$
\begin{gathered}
\left|d_{0}\right\rangle=|0\rangle_{\mathrm{I}}|0\rangle_{\mathrm{II}}|0\rangle_{c} \\
\left|d_{1}\right\rangle=\mathcal{N}\left[\Omega_{\mathrm{II}}(t) g_{\mathrm{I}}|1\rangle_{\mathrm{I}}|0\rangle_{\mathrm{II}}|0\rangle_{c}+\Omega_{\mathrm{I}}(t) g_{\mathrm{II}}|0\rangle_{\mathrm{I}}|1\rangle_{\mathrm{II}}|0\rangle_{c}\right. \\
\left.-\Omega_{\mathrm{I}}(t) \Omega_{\mathrm{II}}(t)|0\rangle_{\mathrm{I}}|0\rangle_{\mathrm{II}}|1\rangle_{c}\right]
\end{gathered}
$$

are eigenstates of the Hamiltonian (2) with zero eigenvalue. Here, $\mathcal{N}$ is a normalization factor and $|0\rangle_{c}\left(|1\rangle_{c}\right)$ is the vacuum state (one-photon state) of the cavity mode. The states $\left|d_{0}\right\rangle$ and $\left|d_{1}\right\rangle$ are dark states since the excited levels $|a\rangle_{\mathrm{I}}$ and $|a\rangle_{\mathrm{II}}$ are unpopulated.

Suppose that the original information carrier is SQUID qubit I, which is in an arbitrary unknown state $\alpha|0\rangle_{\mathrm{I}}+\beta|1\rangle_{\mathrm{I}}$, and SQUID qubit II is initially prepared in the state $|0\rangle$. The cavity mode is in the vacuum state and initial Rabi frequencies satisfy $\Omega_{\mathrm{II}} \gg \Omega_{\mathrm{I}}$. A slow variation of the Rabi frequencies, via adjusting the amplitudes of the pulses, will turn the state $\left(\alpha|0\rangle_{\mathrm{I}}+\beta|1\rangle_{\mathrm{I}}\right)|0\rangle_{\mathrm{II}}|0\rangle_{c}$ into the superposition of the two dark states, i.e., $\alpha\left|d_{0}\right\rangle+$ $\beta\left|d_{1}\right\rangle$. Here and below, the "slow" change is required by adiabatic passage [16]. When slowly changing the Rabi frequencies to $\Omega_{\mathrm{I}} \gg \Omega_{\mathrm{II}}$, one has

$$
\left(\alpha|0\rangle_{\mathrm{I}}+\beta|1\rangle_{\mathrm{I}}\right)|0\rangle_{\mathrm{II}}|0\rangle_{c} \rightarrow|0\rangle_{\mathrm{I}}\left(\alpha|0\rangle_{\mathrm{II}}+\beta|1\rangle_{\mathrm{II}}\right)|0\rangle_{c},
$$

completing QIT from SQUID qubit I to SQUID qubit II.

It is interesting to note the dark-state method can be extended to entangle the two-SQUID qubits. Suppose that the system is initially prepared in $|1\rangle_{\mathrm{I}}|0\rangle_{\mathrm{II}}|0\rangle_{c}$ and that the initial Rabi frequencies satisfy $\Omega_{\mathrm{II}} \gg \Omega_{\mathrm{I}}$. Slowly decreasing $\Omega_{\mathrm{II}}$ while increasing $\Omega_{\mathrm{I}}$ will drive the system to undergo a dark-state evolution described by (5). If the pulses are turned off when $\Omega_{\mathrm{I}}=\Omega_{\mathrm{II}}$, the system will be in the state

$$
\begin{gathered}
\left|d_{1}\right\rangle=\mathcal{N}\left[\Omega_{\mathrm{II}}\left(g_{\mathrm{I}}|1\rangle_{\mathrm{I}}|0\rangle_{\mathrm{II}}+g_{\mathrm{II}}|0\rangle_{\mathrm{I}}|1\rangle_{\mathrm{II}}\right) \otimes|0\rangle_{c}\right. \\
\left.-\Omega_{\mathrm{I}} \Omega_{\mathrm{II}}|0\rangle_{\mathrm{I}}|0\rangle_{\mathrm{II}} \otimes|1\rangle_{c}\right] .
\end{gathered}
$$

Equation (7) implies that if the cavity field is detected in the vacuum state, the two-SQUID qubits are then in the entangled state

$$
\cos \theta|1\rangle_{\mathrm{I}}|0\rangle_{\mathrm{II}}+\sin \theta|0\rangle_{\mathrm{I}}|1\rangle_{\mathrm{II}},
$$

where $\theta=\tan ^{-1}\left(g_{\mathrm{II}} / g_{\mathrm{I}}\right)$. For $g_{\mathrm{I}}=g_{\mathrm{II}}$, we obtain the maximally entangled two-SQUID qubit state

$$
\frac{1}{\sqrt{2}}\left(|1\rangle_{\mathrm{I}}|0\rangle_{\mathrm{II}}+|0\rangle_{\mathrm{I}}|1\rangle_{\mathrm{II}}\right) \text {. }
$$

The cavity-field state can be detected using an auxiliary SQUID initially in the state $|0\rangle$. Set the $|0\rangle \leftrightarrow|a\rangle$ transition resonant with the cavity mode for an interaction time $t_{\text {in }}=\pi /(2 g)$, where $g$ is the coupling constant between the cavity field and the $|0\rangle \leftrightarrow|a\rangle$ transition. One then measures the state of the auxiliary SQUID. If the auxiliary SQUID is found in the level $|0\rangle$, the cavity field was initially in the vacuum state and the two-SQUID qubits I and II are then in the entangled state.

$117902-2$ 
For the method to work, the population of the levels $|a\rangle_{\mathrm{I}}$ and $|a\rangle_{\text {II }}$ must remain zero during the cavity-state measurement and the auxiliary SQUID has to be decoupled from the cavity field during preparing the state (7). This can be met by adjusting the level spacings of the SQUIDs (e.g., by varying $\Phi_{x}$ ). Second, in order to reduce cavity dissipation and spontaneous emission from the level $|a\rangle$, the interaction time $t_{\text {in }}$ for the photon detection should satisfy $t_{\text {in }} \ll \gamma_{a}^{-1}, \kappa^{-1}$ where $\gamma_{a}^{-1}$ is the energy relaxation time of the level $|a\rangle$ and $\kappa^{-1}$ is the photon lifetime of the cavity field. The latter is given by $\kappa^{-1}=Q /\left(2 \pi \nu_{c}\right)$ where $Q$ is the quality factor of the cavity.

To show the scheme is experimentally feasible, consider a SQUID-cavity system with the parameters listed in Table I. Note that SQUIDs with these parameters are readily available at the present time [10-12]. For a superconducting standing-wave cavity and a SQUID located at one of antinodes of the $\mathbf{B}$ field, the coupling constant is $g \sim 1.8 \times 10^{8} \mathrm{~s}^{-1}$. The corresponding interaction time for the single photon detection is $t_{\text {in }}=\pi /(2 g) \sim 8.7 \times$ $10^{-9} \mathrm{~s}$, much smaller than $\gamma_{a}^{-1}$ and $\kappa^{-1} \sim 7.6 \times 10^{-7} \mathrm{~s}$, which is a conservative estimate for superconducting microwave cavities based on recent experiments [17].

The adiabatic passage requires the two applied pulses to be long enough and their areas to be overlapped significantly, but they are not required to have a specific shape or area. Without loss of generality, consider two pulses with Gaussian envelopes

$$
\Omega_{\mathrm{I}}(t)=\Omega_{0} e^{-\left(t-\tau_{\mathrm{I}}\right)^{2} / \Delta \tau^{2}}, \quad \Omega_{\mathrm{II}}(t)=\Omega_{0} e^{-\left(t-\tau_{\mathrm{II}}\right)^{2} / \Delta \tau^{2}},
$$

which were widely used in experiment [18]. The QIT (6) is equivalent to a transformation $|0\rangle_{\mathrm{I}}|0\rangle_{\mathrm{II}}|0\rangle_{c} \rightarrow$ $|0\rangle_{\mathrm{I}}|0\rangle_{\mathrm{II}}|0\rangle_{c}$ and $|1\rangle_{\mathrm{I}}|0\rangle_{\mathrm{II}}|0\rangle_{c} \rightarrow|0\rangle_{\mathrm{I}}|1\rangle_{\mathrm{II}}|0\rangle_{c}$. The state $|0\rangle_{\mathrm{I}}|0\rangle_{\mathrm{II}}|0\rangle_{c}$ remains unchanged due to energy conservation. Thus, to evaluate how closely the proposed system meets the adiabatic passage of the dark states, one needs to investigate to what extent the state $|1\rangle_{\mathrm{I}}|0\rangle_{\mathrm{II}}|0\rangle_{c}$ changes to $|0\rangle_{\mathrm{I}}|1\rangle_{\mathrm{II}}|0\rangle_{c}$. Without losing generality, consider twoSQUID qubits with the same parameters. Plotted in Fig. 2 are results of numerical calculations. Figure 2(b) is for an ideal dark-state evolution described by (5), while Fig. 2(c) is for a full Hamiltonian without the use of rotating-wave approximation. In our calculation, terms describing energy relaxation from levels $|1\rangle$ and $|a\rangle$ of the SQUID qubits as well as the cavity decay are also included in the Hamiltonian. Comparing Figs. 2(b) and 2(c), one can see that the process is an adiabatic passage of dark state (5) since the population on level $|a\rangle$ of each SQUID is less than 0.04 in Fig. 2(c).

Figure 2(c) shows the following instructive QIT and entanglement performance: (i) The population of the state $|0\rangle_{\mathrm{I}}|1\rangle_{\mathrm{II}}|0\rangle_{c}$ is $\sim 0.981$ after a typical time $t=0.3 \mu \mathrm{s}$, thus QIT can be implemented with a high fidelity of $98.1 \%$ for $Q=2 \times 10^{5}$. Increasing quality factor of the cavity to $Q=10^{6}$, which was demonstrated experimentally [17], improves the fidelity to $99.2 \%$. Note that even with a cavity of moderate $Q \simeq 2 \times 10^{4}$ one still achieves good fidelity $(\sim 91.0 \%)$. (ii) For $t \sim 0.21 \mu$ s, i.e., the time when $\Omega_{\mathrm{I}}(t)=\Omega_{\mathrm{II}}(t)$, the cavity field is in the vacuum state with a probability $\sim 0.93$. Moreover, each of the desired states $|1\rangle_{\mathrm{I}}|0\rangle_{\mathrm{II}}|0\rangle_{c}$ and $|0\rangle_{\mathrm{I}}|1\rangle_{\mathrm{II}}|0\rangle_{c}$ is populated with probability $p_{0} \sim 0.435$ while the two unwanted states $|a\rangle_{\mathrm{I}}|0\rangle_{\mathrm{II}}|0\rangle_{c}$ and $|0\rangle_{\mathrm{I}}|a\rangle_{\mathrm{II}}|0\rangle_{c}$ are populated with a small probability $p_{a} \sim$ 0.06 . Thus, the cavity field can be detected in the vacuum state with a high probability $\sim 0.93$ and the two-SQUID qubits can be prepared in the entangled state (9) with a high fidelity $\left[2 p_{0} /\left(2 p_{0}+p_{a}\right)\right] \sim 93.5 \%$. Note that the fidelity could be significantly improved by optimizing the operation time and the system parameters.

The need for making the above cavity-state measurement depends on the type of pulses. For certain types of pulses, such a measurement is not necessary. For instance, if the two pulses satisfy $\Omega_{\mathrm{I}}(t)=\Omega_{\mathrm{II}}(t)$ and $\Omega_{\mathrm{I}}(t)$, $\Omega_{\mathrm{II}}(t) \ll g_{\mathrm{I}}, g_{\mathrm{II}}$ for $t \geq t_{0}$, the entangled state (8) can be created with a probability $P \sim 1$. However, this method requires a precise control of the two pulses so that the two Rabi frequencies $\Omega_{\mathrm{I}}(t)$ and $\Omega_{\mathrm{II}}(t)$ must be kept equal for a significant fraction of the pulse duration.

The scheme works well when direct interaction between the two SQUIDs is negligible. This requirement can be met when $D \gg d$ because the dipole field generated by the current in each SQUID ring at a distance $r \gg$ $d$ decreases as $r^{-3}$. For the cavity mode with $\nu_{c}=$ $41.7 \mathrm{GHz}$ (Table I), the wavelength is $\lambda \sim 7.2 \mathrm{~mm}$. For

TABLE I. Parameters for a SQUID-cavity. $R$ is the SQUID's effective damping resistance. $\gamma_{a}^{-1}\left(\gamma_{1}^{-1}\right)$ is the energy relaxation time of level $|a\rangle(|1\rangle) . \nu_{a 0}\left(\nu_{a 1}\right)$ is the $|0\rangle \leftrightarrow|a\rangle(|1\rangle \leftrightarrow|a\rangle)$ transition frequency. $\phi_{i j} \equiv\langle i|\Phi| j\rangle / \Phi_{0}$ is the coupling matrix element between levels $|i\rangle$ and $|j\rangle(i=a ; j=0,1)$. The cavity has a volume $V$ and length $l . \lambda$ is the wavelength of the cavity mode with frequency $\nu_{c} . \nu_{\mu w l}$ is the carrier frequency of the pulse $l$ with $\Delta \tau$ (full width at half maximum) and $\Omega_{0}$ is the maximum Rabi frequency at the central time $\tau_{l}$ for the pulse $l(l=\mathrm{I}$, II).

\begin{tabular}{|c|c|c|c|c|c|}
\hline SQUID & $\begin{array}{c}C=90 \mathrm{fF} \\
\nu_{a 0}=41.7 \mathrm{GHz}\end{array}$ & $\begin{array}{c}L=100 \mathrm{pH} \\
\nu_{a 1}=33.3 \mathrm{GHz}\end{array}$ & $\begin{array}{c}\beta_{L}=1.14 \\
\phi_{a 0}=5.3 \times 10^{-3}\end{array}$ & $\begin{aligned} \Phi_{x} & =0.4995 \Phi_{0} \\
\phi_{a 1} & =7.3 \times 10^{-3}\end{aligned}$ & $\begin{array}{c}R=65 M \Omega \\
\gamma_{1}^{-1} \sim 100 \mu \mathrm{s} \\
\gamma_{a}^{-1} \sim 2.5 \mu \mathrm{s}\end{array}$ \\
\hline Cavity & $V=7.2 \times 1 \times 1 \mathrm{~mm}^{3}$ & $l=7.2 \mathrm{~mm}$ & $\nu_{c}=41.7 \mathrm{GHz}$ & $\lambda \sim 7.2 \mathrm{~mm}$ & $\begin{array}{c}Q \sim 2 \times 10^{5} \\
\kappa^{-1} \sim 7.6 \times 10^{-7} \mathrm{~s}\end{array}$ \\
\hline Pulse I & $\nu_{\mu w \mathrm{I}}=33.3 \mathrm{GHz}$ & $\Omega_{0}=8.5 \times 10^{7} \mathrm{~s}^{-1}$ & $\tau_{\mathrm{I}}=0.24 \mu \mathrm{s}$ & $\Delta \tau=60 \mathrm{~ns}$ & \\
\hline Pulse II & $\nu_{\mu w \mathrm{II}}=33.3 \mathrm{GHz}$ & $\Omega_{0}=8.5 \times 10^{7} \mathrm{~s}^{-1}$ & $\tau_{\mathrm{II}}=0.18 \mu \mathrm{s}$ & $\Delta \tau=60 \mathrm{~ns}$ & \\
\hline
\end{tabular}



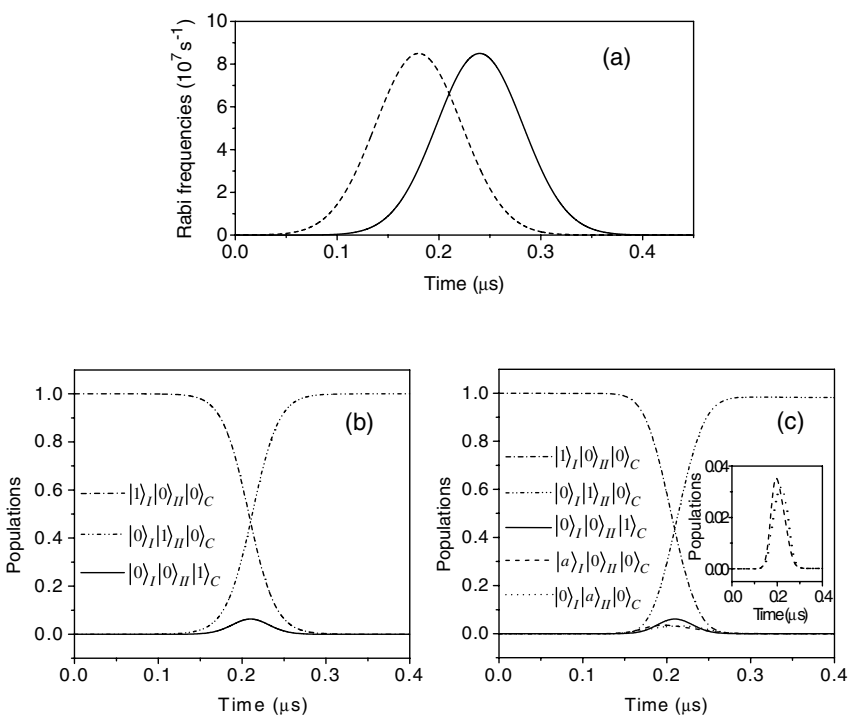

FIG. 2. (a) Rabi frequencies $\Omega_{\text {II }}$ (dash line) and $\Omega_{\mathrm{I}}$ (solid line) versus time. Populations versus time (b) for ideal darkstate evolution, and (c) under the full Hamiltonian. Inset: populations of the states $|a\rangle_{\mathrm{I}}|0\rangle_{\mathrm{II}}|0\rangle_{c}$ (dashed line) and $|0\rangle_{\mathrm{I}}|a\rangle_{\mathrm{II}}|0\rangle_{c}$ (dotted line) versus time. Parameters used for calculations are listed in Table I. The coupling constants used are $g_{\mathrm{I}}=g_{\mathrm{II}}=1.8 \times 10^{8} \mathrm{~s}^{-1}$ (derived from the parameters in Table I).

a cavity with two SQUIDs (I, II) and an auxiliary SQUID at each antinode of the $\mathbf{B}$ field [see Fig. 1(c)], the ratio $D / d \approx 90$ for $d=40 \mu \mathrm{m}$. Thus, the condition of negligible direct coupling is very well satisfied.

Note that implementing QIT via dark states (4) and (5) was first proposed in atomic systems [19]. However, in [19] the coupling constants $g$ of the two qubits are equal for the dark state (5). We find that this requirement is not needed for the dark state (5) because the present QIT protocol is independent of the coupling constants and thus does not require identical SQUID-cavity coupling strength for the two-SQUID qubits. This makes our QIT protocol much easier to implement since neither identical qubits nor exact placement of SQUID qubits in a cavity is needed. Hence, the proposed QIT protocol has the potential of being scaled up because solid state qubits, which often have considerable parameter nonuniformity, can be used. For concreteness, we have presented a set of system parameters (Table I) which are experimentally realizable. Our numerical simulation shows that with these parameters, the proposed process is adiabatic and QIT and entanglement can be achieved with a high fidelity $\sim 1$. Therefore, the present scheme is a significant development in the realization of QIT and entanglement with solid state superconducting devices.

Another very interesting property of our proposal is that the strong coupling limit of cavity QED $g^{2} /(\gamma \kappa) \gg$ 1 , which is difficult to achieve with atoms in a microwave cavity, can be easily realized with SQUID qubits. For SQUIDs, the coupling strength between the qubits and the cavity mode can reach $10^{8} \mathrm{~s}^{-1}$ while for atoms it is on the order of $10^{5} \mathrm{~s}^{-1}$ [20]. Thus, cavity-SQUID systems make the experimental testing of cQED in the strong coupling limit feasible, in addition to providing a new approach for manipulation of microwave photon states, production of nonclassical microwave source, and quantum communication.

This work was supported in part by National Science Foundation (EIA-0082499) and AFOSR (F49620-01-10439), funded under the Department of Defense University Research Initiative on Nanotechnology (DURINT) Program and by the ARDA.

Note added.-After submission of this Letter, a similar work with two identical SQUIDs has been reported recently by Kis and Paspalakis [Phys. Rev. B 69, 024510 (2004)].

[1] Y. Makhlin, G. Schoen, and A. Shnirman, Nature (London) 398, 305 (1999).

[2] Y. Nakamura, Y. Pashkin, and J. S. Tsai, Nature (London) 398, 786 (1999).

[3] A. Steinbach et al., Phys. Rev. Lett. 87, 137003 (2001).

[4] J. M. Martinis and R. L. Kautz, Phys. Rev. Lett. 63, 1507 (1989).

[5] R. Rouse, S. Han, and J. E. Lukens, Phys. Rev. Lett. 75, 1614 (1995).

[6] C. H. van der Wal et al., Science 290, 773 (2000).

[7] S. Han, R. Rouse, and J. E. Lukens, Phys. Rev. Lett. 76, 3404 (1996).

[8] J. R. Friedman et al., Nature (London) 406, 43 (2000).

[9] X. Zhou, J. L. Habif, M. F. Bocko, and M. J. Feldman, quant-ph/0102090.

[10] D. Vion et al., Science 296, 886 (2002).

[11] Y. Yu, S. Han, X. Chu, S.-I. Chu, and Z. Wang, Science 296, 889 (2002).

[12] I. Chiorescu et al., Science 299, 1869 (2003).

[13] Yu. A. Pashkin et al., Nature (London) 421, 823 (2003).

[14] A. J. Berkley et al., Science 300, 1548 (2003).

[15] C. P. Yang, S. I. Chu, and S. Han, Phys. Rev. A 67, 042311 (2003).

[16] J. Oreg, F. T. Hioe, and J. H. Eberly, Phys. Rev. A 29, 690 (1984).

[17] P. K. Day, H. G. LeDuc, B. Mazin, A. Vayonakis, and J. Zmuidzinas, Nature (London) 425, 817 (2003).

[18] T. F. Gallagher, Rydberg Atoms (Cambridge University Press, Cambridge, 1994); U. Gaubatz et al., J. Chem. Phys. 92, 5363 (1990); C. Conover (private communication).

[19] T. Pellizzari, S. A. Gardiner, J. I. Cirac, and P. Zoller, Phys. Rev. Lett. 75, 3788 (1995).

[20] S. Osnaghi et al., Phys. Rev. Lett. 87, 037902 (2001); S. B. Zheng, Phys. Rev. Lett. 87, 230404 (2001); L. You (private communication). 